\title{
Pulmonalvenenablation
}

\section{Cryoballon-Technik eliminiert Vorhofflimmern}

Die Technik der ballongeführten Cryoablation (Arctic Front ${ }^{\mathrm{TM}}$ ) ermöglicht die anatomisch geführte Isolation der Pulmonalvenen mit einer geringen Komplikationsrate.

Die Zahl der Patienten mit Vorhofflimmern (VHF) wird sich in den kommenden Dekaden mehr als verdoppeln. Gleiches gilt auch für die Bedeutung der Ablationstherapie, bei der die Pulmonalvenen mit multiplen punktuellen Hochfrequenzläsionen elektrisch isoliert werden. Die Pulmonalvenenablation hat deutlich bessere Erfolgsraten als die medikamentöse Behandlung, birgt allerdings Komplikationen wie Thromboembolien, Pulmonalvenenstenosen oder Herzbeuteltamponaden als direkte Ablationseffekte sowie atriale Reentry-Tachykardien durch Lücken in der Ablationslinie. Die ballongeführte Cryoab- lation (Arctic Front ${ }^{\mathrm{TM}}$ ) verkürzt potenziell die Lernkurve und reduziert die Gefahr der Lückenbildung mit Reentry-Tachykardien, erklärte Prof. Burghard Schumacher aus Bad Neustadt/Saale. Nach der 3CenterAFStudie lassen sich auf diese Weise akut 97\% aller Lungenvenen isolieren. Eine einzelne Ablation führte bei $74 \%$ der Patienten mit paroxysmalem Vorhofflimmern in der Nachbeobachtungszeit von 18 Monaten zum Sinusrhythmus ohne die Notwendigkeit einer weiteren Medikation.

Die STOP-AF-Studie verglich an $245 \mathrm{~Pa}-$ tienten mit Vorhofflimmern die medikamentöse Therapie ( $n=82)$ und die Cryoablation ( $n=163$ ). Sie belegt nun nach zwölf Monaten Nachbeobachtung eine deutlich höhere Erfolgsrate der Ballonablation $(69,9 \%)$ im Vergleich zur Medikamentengabe (7,3\%). Der Anteil der Patienten mit ablatiertem Vorhofflimmern, die danach noch Antiarrhythmika oder Gerinnungshemmer benötigten, sank von fast 100\% auf etwa $25 \%$. Eine bei $11,2 \%(n=29)$ der ballonbehandelten Patienten aufgetretene Lähmung des Zwerchfellnervs war nach zwölf Monaten bei $86,2 \%(n=25)$ vollständig verschwunden.

In einer Fallstudie mit dem Cryoballon bei 700 Patienten hat Schumacher abgesehen von reversiblen Läsionen des Zwerchfellnervs bei 5,4\% der Patienten keine der für die Radiofrequenzablation typischen Komplikationen beobachtet. Ein weiterer Vorteil war eine deutlich kürzere Durchleuchtungsdauer und die geringere Strahlenbelastung.

(hae) II

Pressekonferenz, Kongress der DGK, Mannheim, April 2010 (Veranstalter: Medtronic)

\section{Prognostischer Vorteil durch Aldosteronantagonisten Herzinsuffizienz-Studie vorzeitig abgebrochen}

\section{Die EMPHASIS-Studie, die den Nutzen des Aldosteronblockers Eplerenon (Inspra ${ }^{\circledR}$ ) bei leichter Herzinsuffizienz untersucht, sollte eigentlich bis Oktober 2011 laufen. Doch das Unter- nehmen Pfizer hat die Rekrutierung weiterer Patienten im Juni vorzeitig eingestellt.}

Bei einer Zwischenanalyse der EMPHASIS-Studie waren die mit einer Standardtherapie plus Eplerenon behandelten Patienten eindeutig im Vorteil: Sie hatten ein signifikant niedrigeres Risiko, aus kardiovaskulärer Ursache zu sterben oder wegen der Herzinsuffizienz stationär eingewiesen zu werden, als Patienten unter einer Standardtherapie plus Placebo.
Die Zusatztherapie mit Eplerenon ist der Zwischenanalyse zufolge auch sicher. Es wurden die von Mineralokortikoidrezeptor-Antagonisten bekannten Nebenwirkungen registriert: Hyperkaliämie (8\% Eplerenon vs. $3 \%$ Placebo) und Niereninsuffizienz ( $4 \%$ vs. $2 \%$ ).

Einschlusskriterium für die EMPHASISStudie war eine chronische systolische Herzinsuffizienz im NYHA-Stadium II. Die Patienten wurden in einem doppelblinden und randomisierten Design 1:1 entweder mit Eplerenon oder Placebo behandelt. Die Einstiegsdosis Eplerenon betrug $25 \mathrm{mg} / \mathrm{d}$, nach vier Wochen konnte - in Abhängigkeit vom Serumkalium - auf $50 \mathrm{mg} / \mathrm{d}$ erhöht werden. Kombinierter primärer Endpunkt war kardiovaskulärer Tod oder Hospitalisie- rung wegen Herzinsuffizienz. Das Studienprotokoll sah eigentlich vor, 3100 Patienten einzubeziehen und die Studie so lange fortzusetzen, bis 813 Ereignisse erreicht waren.

Bisher ist Eplerenon zur Behandlung der chronischen Herzinsuffizienz nicht zugelassen. Die Indikation beschränkt sich vielmehr auf Patienten die nach einem akuten Herzinfarkt eine linksventrikuläre Dysfunktion und/oder Zeichen einer Herzinsuffizienz aufweisen. Bei ihnen wird durch eine frühzeitige und dauerhafte Eplerenonbehandlung die Sterblichkeit signifikant reduziert.

(BS) II

II Pressemitteilung Pfizer, 8. Juni 2010 